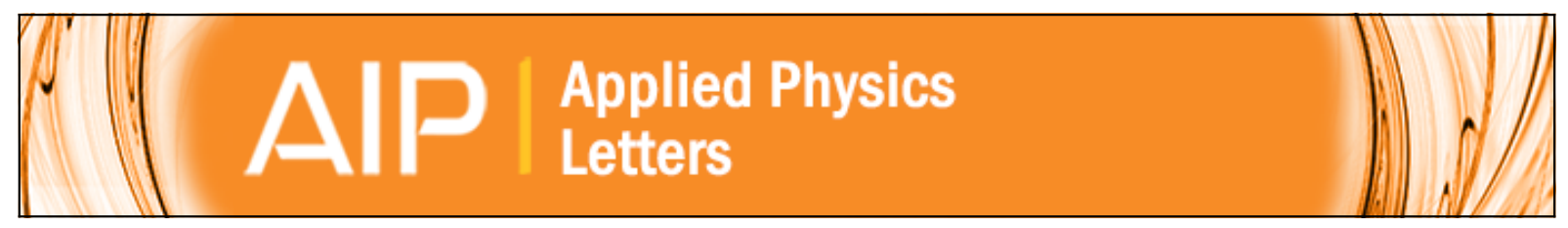

\title{
Effects of deep levels on transconductance dispersion in AIGaAs/InGaAs pseudomorphic high electron mobility transistor
}

Kyoung Jin Choi, Jong-Lam Lee, and Hyung Mo Yoo

Citation: Applied Physics Letters 75, 1580 (1999); doi: 10.1063/1.124760

View online: http://dx.doi.org/10.1063/1.124760

View Table of Contents: http://scitation.aip.org/content/aip/journal/apl/75/11?ver=pdfcov

Published by the AIP Publishing

\section{Articles you may be interested in}

Anomaly and defects characterization by I-V and current deep level transient spectroscopy of Al0.25Ga0.75N/GaN/SiC high electron-mobility transistors

J. Appl. Phys. 111, 073713 (2012); 10.1063/1.3702458

Depletion- and enhancement-mode In $0.49 \mathrm{Ga} 0.51 \mathrm{P} / \mathrm{InGaAs} / \mathrm{AlGaAs}$ high-electron-mobility transistors with high-breakdown voltage

J. Vac. Sci. Technol. B 21, 981 (2003); 10.1116/1.1568354

Surface states on n-type Al 0.24 Ga 0.76 As characterized by deep-level transient spectroscopy

J. Vac. Sci. Technol. B 19, 615 (2001); 10.1116/1.1368679

Application of nonalloyed PdGe ohmic contact to self-aligned gate AIGaAs/InGaAs pseudomorphic high-electronmobility transistor

Appl. Phys. Lett. 74, 2866 (1999); 10.1063/1.124040

Hot carrier effects in AIGaAs/InGaAs high electron mobility transistors: Failure mechanisms induced by hot carrier testing

J. Appl. Phys. 82, 5547 (1997); 10.1063/1.366413

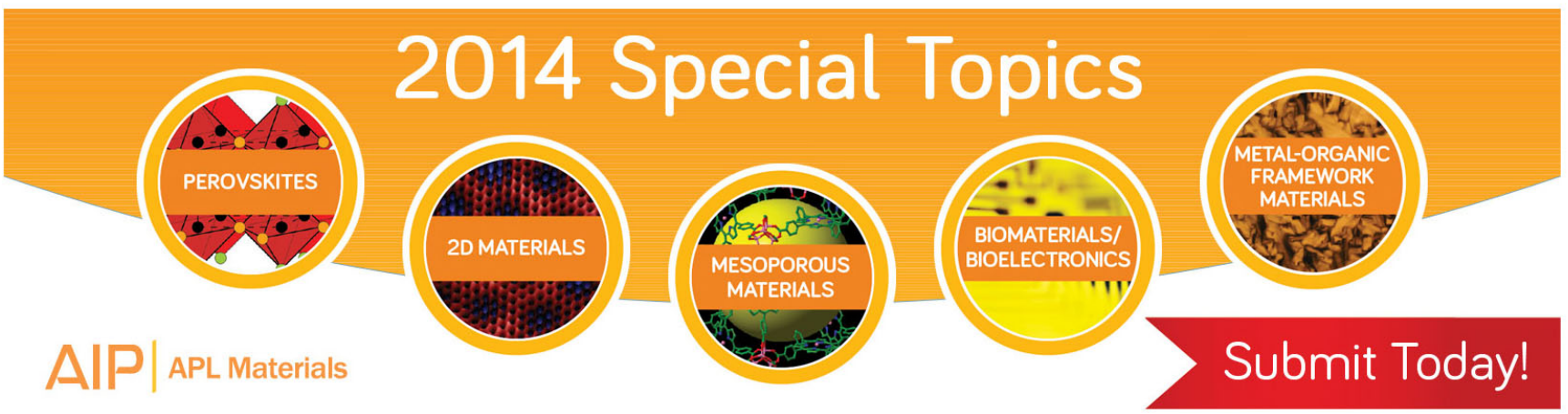




\title{
Effects of deep levels on transconductance dispersion in AIGaAs/InGaAs pseudomorphic high electron mobility transistor
}

\author{
Kyoung Jin Choi and Jong-Lam Lee ${ }^{\text {a) }}$ \\ Department of Materials Science and Engineering, Pohang University of Science and Technology \\ (POSTECH), Pohang, Kyungbuk 790-784, Korea
}

Hyung Mo Yoo

Samsung Microwave Semiconductor, 1530 McCarthy Boulevard, Milpitas, California 95035

(Received 28 April 1999; accepted for publication 19 July 1999)

\begin{abstract}
The effects of deep levels on the transconductance dispersion in an AlGaAs/InGaAs pseudomorphic high electron mobility transistor was interpreted using capacitance deep level transient spectroscopy (DLTS). Transconductance was decreased by $10 \%$ in the frequency range of $10 \mathrm{~Hz}-10 \mathrm{kHz}$ at the negative gate bias, but it was increased at the positive one. In the DLTS spectra, two hole trap-like signals corresponding to surface states were only observed at the negative pulse bias, whereas the $D X$-center with the activation energy of $0.42 \pm 0.01 \mathrm{eV}$ were observed at the positive one. The activation energy agrees well with that obtained from the temperature dependence of the positive transconductance dispersion, $0.39 \pm 0.03 \mathrm{eV}$. These provide evidence that the positive and negative transconductance dispersions are due to the $D X$ center and surface states, respectively. (c) 1999 American Institute of Physics. [S0003-6951(99)04437-X]
\end{abstract}

$\mathrm{AlGaAs} / \mathrm{InGaAs}$ pseudomorphic high electron mobility transistors (PHEMTs) have been successful in a variety of applications, including high performance low-noise amplifiers and monolithic power amplifiers. In the PHEMT, the extrinsic transconductance exhibited significant frequency dispersion at frequencies lower than $1 \mathrm{MHz}$. The transconductance dispersion caused the undesirable characteristics, such as the hysteresis in $I-V$ curve, ${ }^{1}$ the low breakdown voltage behavior, ${ }^{2}$ and the increase in the low frequency noise. $^{3}$

The origin of the transconductance dispersion has been studied using a variety of measurement techniques. The transconductance of PHEMTs was decreased (negative transconductance dispersion) and/or increased (positive transconductance dispersion) with frequency. ${ }^{4,5}$ The negative transconductance dispersion, commonly observed in GaAs metalsemiconductor field-effect transistors, was believed to be due to the surface states existing on the ungated regions between gate and source/drain electrodes. ${ }^{1,6,7}$ The magnitude of the negative transconductance dispersion was dependent on the surface leakage current from the gate ${ }^{1}$ and could be reduced after the surface passivation with $\mathrm{Si}_{3} \mathrm{~N}_{4} \cdot{ }^{6}$ On the other hand, the positive transconductance dispersion was observed in HEMT with the source layer of Si-doped AlGaAs, which was thought to be due to bulk traps, such as $D X$ center and point defects produced by the neutron irradiation. ${ }^{8}$ In the deep-level transient spectroscopy (DLTS) of HEMT structures, the $D X$-center peak with the activation energy of 0.43 $\mathrm{eV}^{9,10}$ was often observed. Several works have been independently conducted on the $D X$ center using DLTS $^{9}$ and the transconductance dispersion measurements. ${ }^{5}$ There was no work to show the direct relationship between the $D X$ center and the transconductance dispersion. In PHEMTs with short

${ }^{a)}$ Electronic mail: jllee@ vision.postech.ac.kr gate periphery, the change of capacitance produced by the $D X$ center was too small to be detected in capacitance transients. Provided that AlGaAs/InGaAs PHEMTs with large gate periphery are examined using the capacitance DLTS, one could obtain the information for deep levels and clearly interpret their effects on the transconductance dispersion.

In the present work, capacitance DLTS and transconductance dispersion measurements are simultaneously performed on an $\mathrm{AlGaAs} / \mathrm{InGaAs}$ PHEMT with large gate periphery. The applied pulse bias dependence of both the DLTS spectrum and transconductance dispersion profile are compared with each other. From this, the type of trap causing the transconductance dispersion in the $\mathrm{AlGaAs} / \mathrm{InGaAs}$ PHEMT is determined, and origins of positive and negative transconductance dispersions will be discussed.

The layer structure of a PHEMT was prepared by molecular-beam-epitaxy on a semiinsulating GaAs substrate, as shown in Fig. 1. The buffer layers consist of a $0.6-\mu \mathrm{m}-$

\begin{tabular}{|c|c|}
\hline Source & Drain \\
\hline $\mathrm{n}^{+}-\mathrm{GaAs}$ & $350 \AA$ \\
\hline $\mathrm{n}-\mathrm{GaAs}$ & $200 \AA$ \\
\hline $\mathrm{n}-\mathrm{Al}_{0.24} \mathrm{GaAs}, 2.0 \times 10^{17} / \mathrm{cm}^{3}$ & $250 \AA$ \\
\hline \multicolumn{2}{|l|}{ Si planar doping, $5.0 \times 10^{12} / \mathrm{cm}^{2}$} \\
\hline Undoped $\mathrm{Al}_{0.24} \mathrm{GaAs}$ spacer & $50 \AA$ \\
\hline Undoped $\mathrm{In}_{0.22} \mathrm{GaAs}$ channel & $125 \AA$ \\
\hline Undoped $\mathrm{Al}_{0.24} \mathrm{GaAs}$ spacer & $50 \AA$ \\
\hline $\mathrm{n}-\mathrm{Al}_{0.24} \mathrm{GaAs}, 9.0 \times 10^{17} / \mathrm{cm}^{3}$ & $50 \AA$ \\
\hline $\mathrm{GaAs} / \mathrm{Al}_{0.24} \mathrm{GaAs}$ superlattice & 12 periods \\
\hline Undoped $\mathrm{GaAs}$ buffer & $6000 \AA$ \\
\hline
\end{tabular}

FIG. 1. The cross-sectional diagram of the AlGaAs/InGaAs PHEMT used in this work. 


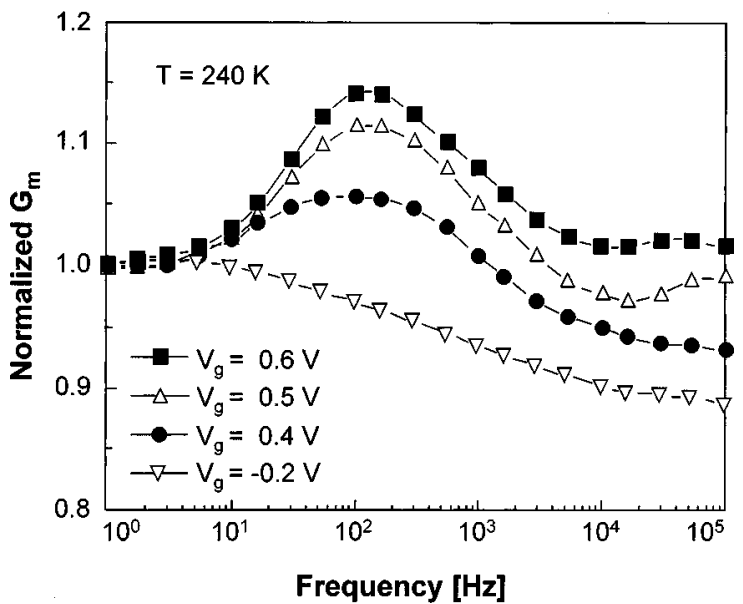

FIG. 2. The transconductance dispersion of the AlGaAs/InGaAs PHEMT as a function of the gate bias. Drain bias was kept at $+0.5 \mathrm{~V}$.

thick undoped GaAs layer and a 12-period undoped $\mathrm{Al}_{0.24} \mathrm{Ga}_{0.76} \mathrm{As} / \mathrm{GaAs}$ superlattice. The active part of the structure is a 125 - $\AA$-thick $\operatorname{In}_{0.22} \mathrm{Ga}_{0.78} \mathrm{As}$ channel layer sandwiched between upper and lower 50 - $\AA$-thick $\mathrm{Al}_{0.24} \mathrm{Ga}_{0.76} \mathrm{As}$ spacer layers. The two-dimensional electron gas (2DEG) is formed in the pseudomorphic InGaAs channel by electron transfer from upper silicon-planar-doped layer with a density of $5 \times 10^{12} / \mathrm{cm}^{2}$ and from the lower $n$-type $\mathrm{Al}_{0.24} \mathrm{Ga}_{0.76} \mathrm{As}$ layer with a density of $9 \times 10^{17} / \mathrm{cm}^{3}$. An $n$-type $\mathrm{Al}_{0.24} \mathrm{Ga}_{0.76} \mathrm{As}$ layer was grown on the planar-doped layer for Schottky contact. Finally, double layers of $n$-type GaAs cap were adopted to provide both good ohmic contacts and high breakdown voltage. PHEMTs with a total gate width of 1.5 mm composed of ten fingers, each with a width of $150 \mu \mathrm{m}$ were fabricated on the substrate. The gate length was $1 \mu \mathrm{m}$, and gate-to-source and gate-to-drain spacings were 0.5 and $1.1 \mu \mathrm{m}$, respectively. The PHEMTs were loaded into a variable-temperature cryostat, and capacitance DLTS measurements were made using a $1 \mathrm{MHz}$ capacitance meter, a pulse generator, and a temperature controller. The temperature of the device was cooled down below $90 \mathrm{~K}$ using liquid nitrogen and heated until $400 \mathrm{~K}$.

For the measurement of transconductance, namely, the ratio of incremental change of drain current to an incremental change of gate voltage, a small sinusoidal signal was applied to the gate of the device through the matching circuit, and the corresponding sinusoidal current was measured at the drain side of the PHEMT through the load resistor using an oscilloscope. The transconductance was measured in a frequency range of $1-10^{5} \mathrm{~Hz}$ and the peak-to-peak value of the applied sinusoidal wave was kept to $100 \mathrm{mV}$.

Current-voltage characteristics of the AlGaAs/InGaAs PHEMT was measured by pulse signal with a period of 0.1 $\mathrm{ms}$. The pinchoff voltage was measured to be $-0.6 \mathrm{~V}$. The maximum drain current, measured at $V_{g s}=+1.0 \mathrm{~V}$, is 0.76 $\mathrm{A}$ and its density is $507 \mathrm{~mA} / \mathrm{mm}$. Figure 2 shows the transconductance dispersion of the PHEMT as a function of the gate bias. The results were normalized to the value measured at $1 \mathrm{~Hz}$. The gate bias was changed from -0.2 to $+0.6 \mathrm{~V}$ at the constant drain bias of $+0.5 \mathrm{~V}$. The negative transconductance dispersion is observed in the broad frequency range of $5.5-1.7 \times 10^{4} \mathrm{~Hz}$ at a negative gate bias of $-0.2 \mathrm{~V}$. As the

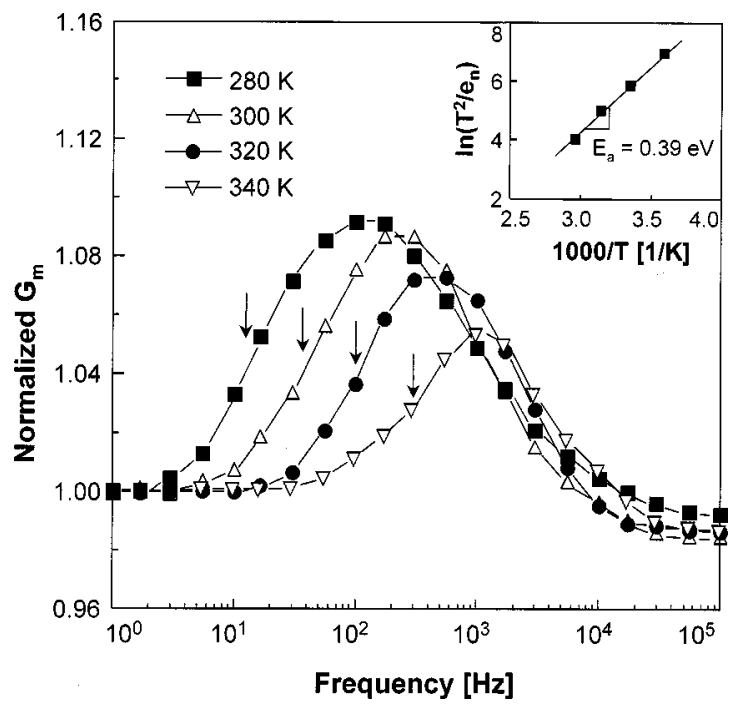

FIG. 3. The transconductance dispersion of the AlGaAs/InGaAs PHEMT as a function of device temperature. The inset shows the temperature dependence of transition frequencies obtained in the positive transconductance. Gate and drain biases were kept at $+0.5 \mathrm{~V}$.

gate bias becomes positive, transconductance is increased with the frequency, followed by decreased in the frequency range of 5.5-3000 Hz. The magnitude of the positive transconductance dispersion becomes more prominent with the increase of the gate bias.

Figure 3 shows the transconductance dispersion of the PHEMT as a function of temperature. The gate and drain biases were fixed to be $+0.5 \mathrm{~V}$. The frequency corresponding to half value in the increase of transconductance was defined as the transition frequency, which is marked by an arrow in Fig. 3. The transition frequency shifts to higher frequencies with the increase of device temperature. The shift of the transition frequency in Fig. 3 is directly related to the change of the electron emission rate with device temperature because the emission rate is identical with a reciprocal of the transition frequency. Thus, one can determine the activation energy for the emission of electrons from the bulk electron trap, which is determined to be $0.39 \pm 0.03 \mathrm{eV}$, as shown in the inset of Fig. 3.

Capacitance DLTS measurements were conducted on the same PHEMT to determine the type of trap causing the transconductance dispersion in the PHEMT. Figure 4 displays the DLTS spectra of the PHEMT. The pulse bias $\left(V_{p}\right)$ applied to the gate was changed in the range of -0.1 to +0.6 $\mathrm{V}$, keeping a constant negative bias $\left(V_{m}\right)$ of $-0.8 \mathrm{~V}$. The $V_{m}$ is slightly lower than the pinchoff voltage of the PHEMT, $-0.6 \mathrm{~V}$. Thus, DLTS signals observed originate from the point defects in the active layers above superlattice (see Fig. 1). The DLTS signal corresponds to the difference between capacitances measured at different sampling times, namely $C\left(t_{1}\right)-C\left(t_{2}\right)$. Thus, the positive signal in the DLTS spectrum corresponds to a hole trap, and the negative one is an electron trap. Two types of hole trap-like signals are observed at $240 \mathrm{~K}(\mathrm{H} 1)$ and $340 \mathrm{~K}(\mathrm{H} 2)$, and an electron trap is observed at $190 \mathrm{~K}(\mathrm{E} 1)$. Heights of $\mathrm{H} 1$ and $\mathrm{H} 2$ peaks are constant, but that of E1 is increased with the increase of $V_{p}$. The activation energies and the capture cross sections for these traps were determined from the temperature dependen- 


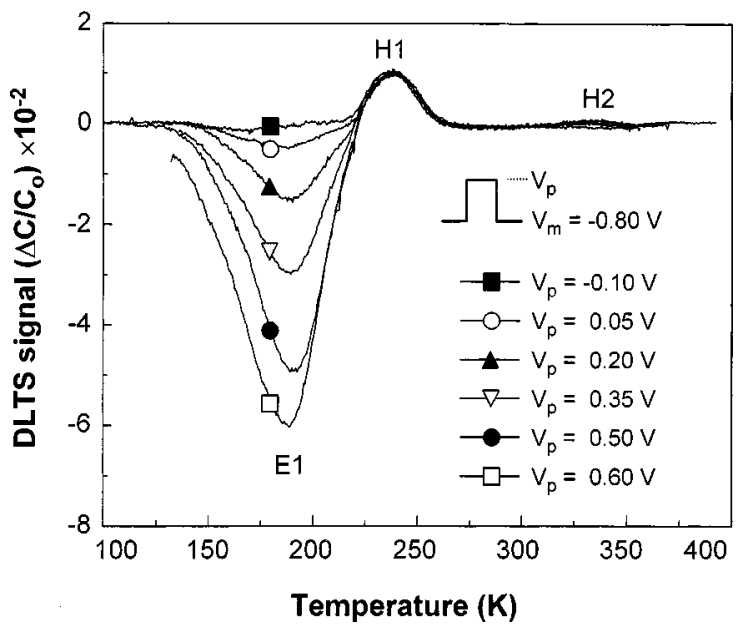

FIG. 4. The DLTS spectra of the AlGaAs/InGaAs PHEMT as a function of $V_{p}$.

cies of $\mathrm{H} 1, \mathrm{H} 2$, and $\mathrm{E} 1$, summarized in Table I.

When the $\mathrm{Al}_{x} \mathrm{Ga}_{1-x} \mathrm{As}(x>0.23)$ layer is doped with $n$-type dopants, the $D X$ center, known as the complex of the substitutional donor atom with an unknown lattice defect, are generated. The activation energy of E1 peak, 0.42 $\pm 0.01 \mathrm{eV}$, agrees well with the previously reported value of the $D X$ center, $0.43 \mathrm{eV} .{ }^{9,10}$ This suggests that the $\mathrm{E} 1$ peak is from the $D X$ center. Even though hole injection is not available in the $n$-type device, two hole trap-like signals were observed. The hole-like signals are related to the surface states at the ungated regions of the device between gate and source/drain electrodes. ${ }^{6}$

The $D X$-center peak is decreased with the decrease of $V_{p}$ and disappears at $V_{p}$ smaller than $-0.1 \mathrm{~V}$. This means that the energy level of the $D X$ centers, $E_{D X}$, becomes higher (or lower) than the quasi-Fermi level of electrons, $E_{F n}$, and the $D X$ centers are free from (or filled with) electrons. This results in the disappearance (or generation) of the $D X$-center peak in DLTS spectra at $V_{p}$ smaller (or larger) than $-0.1 \mathrm{~V}$. As $V_{p}$ is increased, more electrons are captured by the $D X$ center, via increase of the $D X$-center peak, as shown in Fig. 4.

Comparing the results of the transconductance dispersion with those of DLTS measurements, the positive transconductance dispersion and the $D X$-center peak in the DLTS spectra were only observed at positive gate biases and their amplitudes were increased with the increase of the gate biases. These provide evidence that the $D X$ center causes the positive transconductance dispersion in a PHEMT. ${ }^{4}$ Namely, at frequencies lower than the characteristic frequency of electrons in the $D X$ center, electrons are able to follow the applied sinusoidal signal and are fully modulated. During the positive (or negative) semiperiod of the sinusoidal signal,

TABLE I. The activation energies and the capture cross sections for $\mathrm{H} 1, \mathrm{H} 2$, and E1 traps.

\begin{tabular}{ccc}
\hline \hline Type of trap & Activation energy $(\mathrm{eV})$ & Capture cross section $\left(\mathrm{cm}^{2}\right)$ \\
\hline H1 & $0.50 \pm 0.03$ & $2.4 \times 10^{-14}$ \\
H2 & $0.81 \pm 0.01$ & $3.4 \times 10^{-13}$ \\
E1 & $0.42 \pm 0.01$ & $1.5 \times 10^{-14}$ \\
\hline \hline
\end{tabular}

$E_{D X}$ becomes lower (or higher) than $E_{F n}$, and thus electrons are captured by (emitted from) $D X$ centers. This results in the reduction of the modulation of the 2DEG in the InGaAs channel, via the lower value of transconductance. At frequencies higher than the characteristic frequency, electrons in $D X$ centers are, however, unable to follow the sinusoidal signal. Thus, the sinusoidal signal applied to the gate fully modulates the 2DEG, resulting in the increase of transconductance.

The negative transconductance dispersion in Fig. 2, observed at the negative gate bias of $-0.2 \mathrm{~V}$, is interpreted to be related to the surface states at the ungated region between gate and source/drain electrodes. ${ }^{1,6,7}$ At frequencies lower than the characteristic frequency of electrons in surface states, electrons occupied in the surface states are able to follow the applied sinusoidal signal. Thus, depletion layers under the ungated and gated regions are simultaneously modulated. At frequencies higher than the characteristic frequency, the electrons are not, however, able to follow the signal, and the depletion region under the gate is only modulated, resulting in the decrease of the transconductance, as shown in Fig. 2.

In conclusion, the transconductance dispersion in a AlGaAs/InGaAs PHEMT was interpreted using capacitance DLTS measurements. Transconductance was decreased with frequency at negative bias of gate, but it was increased at the positive bias. The activation energy obtained from the temperature dependence of the transition frequency was determined to be $0.39 \pm 0.03 \mathrm{eV}$. In the capacitance DLTS measurements, $D X$ center with activation energy of 0.420 $\pm 0.01 \mathrm{eV}$ and two surface states with $0.50 \pm 0.03$ and 0.81 $\pm 0.01 \mathrm{eV}$ were observed. The activation energy of the $D X$ center agrees well with that for the positive transconductance dispersion. And the gate-bias dependencies of the positive transconductance dispersion and the $D X$-center peak in the DLTS spectra were consistent with each other. The results suggest that the $D X$ center and surface state cause the positive and negative transconductance dispersions in the AlGaAs/InGaAs PHEMT.

This work was financially supported by the Ministry of Information and Communication, Korea, under Contract No. 98-229.

${ }^{1}$ M. Ozeki, K. Kodama, M. Takikawa, and A. Shibatomi, J. Vac. Sci. Technol. 21, 438 (1982).

${ }^{2}$ J.-L. Lee, D. Kim, S. F. Maeng, H. H. Park, J. Y. Kang, and Y. T. Lee, J. Appl. Phys. 73, 3539 (1993).

${ }^{3}$ Y.-J. Chan and D. Pavlidis, IEEE Trans. Electron Devices ED-41, 637 (1994).

${ }^{4}$ W. Kruppa and B. Boos, IEEE Trans. Electron Devices ED-44, 687 (1997).

${ }^{5}$ G. Meneghesso, E. D. Bortoli, D. Sala, and E. Zanoni, Microelectron. Reliab. 37, 1121 (1997).

${ }^{6}$ P. H. Ladbrooke and S. R. Blight, IEEE Trans. Electron Devices ED-35, 257 (1988).

${ }^{7}$ J. H. Zhao, P. F. Tang, R. Hwang, and S. Chang, J. Appl. Phys. 70, 1899 (1991).

${ }^{8}$ G. Meneghesso, A. Paccagnella, D. V. Camin, N. Fedyakin, G. Pessina, and C. Canali, IEEE Trans. Nucl. Sci. 44, 840 (1997).

${ }^{9}$ L. Lu, S. Feng, J. Liang, Z. Wang, J. Wang, Y. Wang, and W. Ge, J. Cryst. Growth 169, 637 (1996).

${ }^{10}$ J. Woeckinger, W. Jantsch, Z. Wilamowski, and K. Koehler, J. Appl. Phys. 74, 1051 (1993). 